\title{
Prediction of Far-Field Pattern Characteristics of Phased Array Fed Reflector Antennas By Modeling Only a Small Part of the Array - Case Study of Spaceborne Radiometer Antennas
}

\author{
O. A. Iupikov*, A. A. Roev*, M. V. Ivashina* \\ *Chalmers University of Technology, Göteborg, Sweden, oleg.iupikov@chalmers.se, roev@chalmers.se, \\ marianna.ivashina@chalmers.se
}

\begin{abstract}
In this work we present an approach of simplified analysis of focal plane array (FPA) systems, in which phasedshifted versions of the simulated embedded element pattern (EEP) of the center element are used to compose a set of all EEPs of the full-scale array and thereafter combined with the optimum weighting coefficients in order to find the total pattern of the feed. Although, the EEPs of dense array antennas are generally not identical (due to the array antenna mutual coupling and edge truncation effects), for typical FPA excitation scenarios, where the array edge elements have relatively low weights to produce the desired illumination of the reflector, this simplified approach has been found sufficiently accurate.
\end{abstract}

Index Terms-reflector antenna feeds, array antennas, microwave radiometers

\section{INTRODUCTION}

Recent advances in radio-frequency and digital electronics have allowed for the design of novel antenna systems, which have superior beamforming capabilities. Examples of such systems are spaceborne antennas for ocean surveillance and satelitte communication; they are required to provide multiple high-efficiency beams (with extremely low side-lobes or crosspolarization) and operate at several frequency bands (typically L-, C-, X and Ku-bands), while having a compact singleantenna design. These challenging requirements can be met by using dense focal plane arrays (FPAs) feeding a reflector (or a lens), or directly-radiating sparse irregular arrays [1], [2]. However, there are common problems with such large and multi-scale antenna designs, including fast and accurate electromagnetic analysis as well as cost-efficient prototype development. Different approaches have been proposed to overcome these problems for the sparse arrays, where performance of the whole antenna system is evaluated through the analysis of a small part of it (e.g. [1], [2]).

In this work we address this problem for the case of FPA systems, and in particular present a validated simplified approach where a reduced-size FPA simulations are used to predict the performance of the whole array feeding the reflector antenna.

\section{ANTENNA GEOMETRY AND SPECIFICATIONS}

To demonstrate the proposed approach we have considered a conical-scan offset parabolic reflector antenna (projected aperture diameter is $5 \mathrm{~m}$, focal length is $3 \mathrm{~m}$ and clearance is $1 \mathrm{~m}$ ) with the 67-element array feed. This antenna system is currently being considered for potential future ocean missions by ESA [3]. The requirements for this mission are given in Table I, [4], in terms of standard performance metrics for oceanographic surveys. For the given satellite altitude and incidence angle, the radiometric requirements can be transfered [5] to the antenna system specifications as shown in Table II.

TABLE I

RADIOMETRIC REQUIREMENTS FOR FUTURE OCEAN MISSIONS

\begin{tabular}{|c|c|c|c|c|c|c|}
\hline $\begin{array}{c}\text { Freq., } \\
{[\mathrm{GHz}]}\end{array}$ & $\begin{array}{c}\text { Band } \\
\text { width, } \\
{[\mathrm{MHz}]}\end{array}$ & $\begin{array}{c}\text { Polari- } \\
\text { zation }\end{array}$ & $\begin{array}{c}\text { Radiometric } \\
\text { resolution, } \\
{[\mathrm{K}]}\end{array}$ & $\begin{array}{c}\text { Bias, } \\
{[\mathrm{K}]}\end{array}$ & $\begin{array}{c}\text { Spatial } \\
\text { resolution, } \\
{[\mathrm{km}]}\end{array}$ & $\begin{array}{c}\text { Dist.to } \\
\text { coast, } \\
{[\mathrm{km}]}\end{array}$ \\
\hline $\begin{array}{c}\text { L-band: } \\
1.404- \\
1.423\end{array}$ & 19 & $\mathrm{~V}, \mathrm{H}$ & 0.15 & 0.25 & 100 & $\begin{array}{c}50- \\
100\end{array}$ \\
\hline $\begin{array}{c}\text { C-band: } \\
6.8-7.0 \\
7.2-7.4\end{array}$ & 200 & $\mathrm{~V}, \mathrm{H}$ & 0.30 & 0.25 & 20 & $15-20$ \\
\hline
\end{tabular}

TABLE II

ANTENNA REQUIREMENTS

\begin{tabular}{|l|c|c|}
\hline Antenna characteristic & L-band & C-band \\
\hline Number of beams & 4 & 2 \\
\hline Cross-polar. power over the Earth & \multicolumn{2}{|c|}{$<0.34 \%$} \\
\hline Power over the land & $<0.28 \%$ \\
\hline Projected aperture diameter of the reflector & \multicolumn{2}{|c|}{$5 \mathrm{~m}$} \\
\hline
\end{tabular}

In previous system-level studies, we have applied this simplified approach to cross-compare different radiometer system concepts, i.e. a traditional conical-scan off-set parabolic reflector antenna vs. a wide-scan torus reflector system [6], [7], as well as to perform parametric studies for the FPAs to define the optimal number of antenna elements, inter element spacing, and the arrangement of FPAs operating at different bands [6], [8], [9]. In the current work, we validate this approach for the 
case of a wideband Vivaldi antenna element FPA feeding the conical-scan reflector antenna, and use for this purpose the requirements in Table I. To simplify the prototyping phase, our focus will be on the high frequency performance only $(\mathrm{C}$ band), for which the small-size array demonstrator has only 24 elements, while the operational bandwidth of the designed full-scale array covers both L- and C-bands.

\section{ARRAY ANTENNA DESIGN}

The Vivaldi antenna element in [10], which most closely meets the wide-band requirements of the project, was chosen as a reference: it has the relative bandwidth greater than 6:1 over wide scan range $( \pm 45 \mathrm{deg})$. Since the geometry of the referred TSA in [10] is for the frequency band of $0.4-1.6 \mathrm{GHz}$, we have scaled up this design with some modifications related to the following practical implementation aspects:

- to improve the mechanical stability;

- to improve the matching for the reference impedance of $50 \mathrm{Ohm}$ (in opposite to the original design, where 70 Ohm LNAs are used).

Thus a new element geometry of a dual-polarized phased array has been optimized and analyzed with the aid of periodic boundary conditions. The slotline width, rate of exponential slotline, cavity length, stub radius and stripline width were chosen as variable parameters. The main goal was to achieve the impedance matching condition with magnitude of the active reflection coefficient less than $-10 \mathrm{~dB}$ within $\pm 45 \mathrm{deg}$ scan range. The optimization have been performed with the commercially available EM software HFSS and CST.

The final antenna and feed geometries with dimensions are shown in Fig. 1(a) and 1(b), respectively. Tapered slot profile is determined by curve:

$$
y=C_{1} e^{R x}+C_{2},
$$

where $R$ is the rate of exponential slotline, and coefficients $C_{1}$ and $C_{2}$ are defined as

$$
\begin{aligned}
C_{1} & =\frac{y_{2}-y_{1}}{e^{R x_{2}}-e^{R x_{1}}} \\
C_{2} & =\frac{y_{1} e^{R x_{2}}-y_{2} e^{R x_{1}}}{e^{R x_{2}}-e^{R x_{1}}},
\end{aligned}
$$

where points $\left(x_{1}, y_{1}\right)$ and $\left(x_{2}, y_{2}\right)$ determine a slot width in the excitation region and the aperture, respectively.

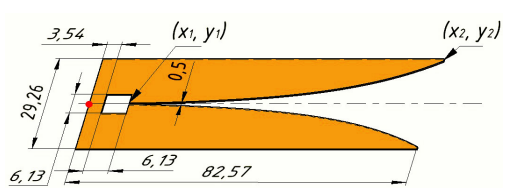

(a)

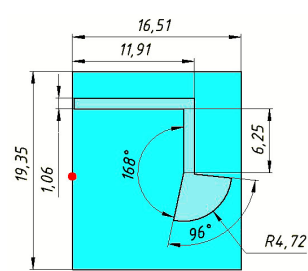

(b)

\begin{tabular}{|c|c|c|c|c|}
\hline$x_{1}$ & $y_{1}$ & $x_{2}$ & $y_{2}$ & $R$ \\
\hline 13.21 & 0.25 & 82.57 & 13.92 & 0.04 \\
\hline
\end{tabular}

Fig. 1. Geometrical dimensions of (a) the proposed TSA element and (b) feeding plate. All dimensions are given in $[\mathrm{mm}]$.
Based on the simulations, a prototype of the small-scale dual-polarized array, comprising 24 elements, was designed and manufactured (Fig. 2). The array antenna structure consists of 4 orthogonally placed brass sheets with 3 TSA elements per polarization. All elements are mounted on the 250x250 mm aluminum ground plane. Each element is excited directly by a PCB feed with the SMA connector located under the ground plane.

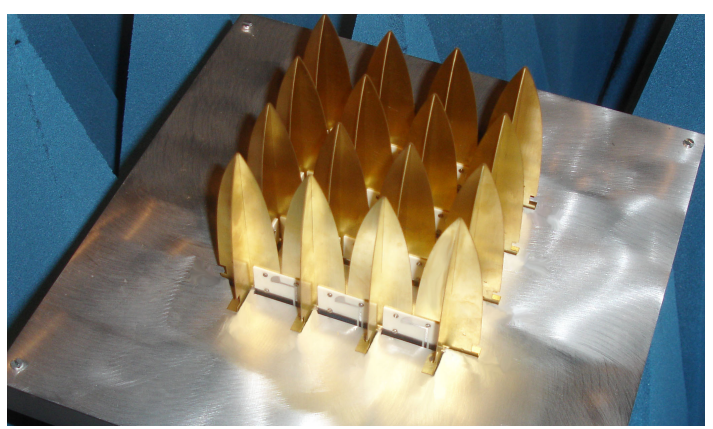

Fig. 2. Photo of the manufactured reduced prototype.

\section{ANALYSIS METHODOLOGY AND NUMERICAL RESULTS}

For typical FPA excitation scenarios, the antenna elements at the edge of the array have significantly $(-5 \ldots-15 \mathrm{~dB})$ lower weighting coefficients relatively to the elements in the center. This implies that the differences in the embedded element pattern shapes, introduced by the edge effects, will have relatively weak contribution to the total compound beam of the array when all elements are excited. This motivates our assumption on the identical EEPs that can be taken to be the same as the pattern of an element in the center. Such approach can greatly speed up the numerical analysis of a reflector antenna system, which is very important for optimization.

The antenna specifications (see Table II) define the required array layout and aperture area, which are shown in Fig. 3(a). In order to validate the proposed analysis approach, we have used the full-wave simulation results for this array as the reference for the following simplified models:

1) Simplified model I, where FPA EEPs are phase-shifted versions of the EEP of the central element (element No.18), which was obtained for the full-scale array;

2) Simplified model II, where FPA EEPs are phase-shifted versions of the EEP of the central element (element No.5), which was obtained for the small-sized array, shown in Fig. 3(b).

Figure 4 shows the EEPs for all these cases.

Figure 3(c) shows the weighting coefficients for Simplified models I and II have been found through the dedicated optimum beamforming procedure detailed in [11] that aims to satisfy the radiometric requirements. The coefficients for the small-sized array have been chosen to be a sub-set of the calculated coefficients that correspond to the most strongly excited elements; they are shown in Fig. 3(d). 


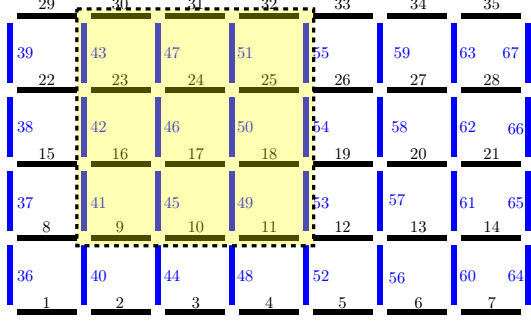

(a)

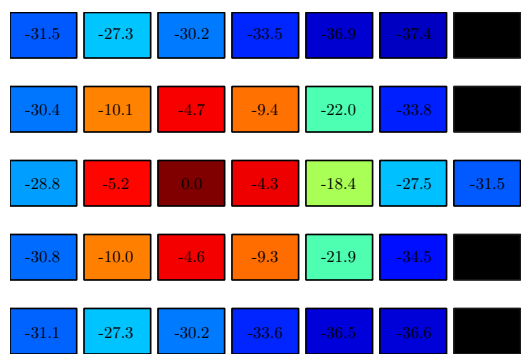

(c)

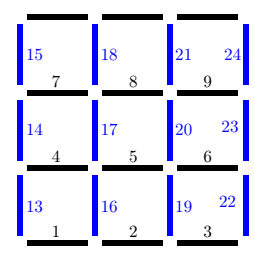

(b)

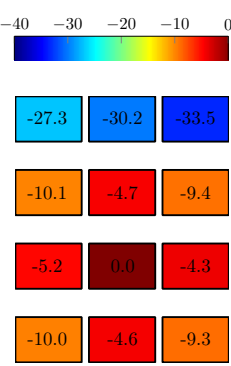

(d)
Fig. 3. (a,c) Full-size array and (b,d) small-sized array layouts, and the corresponding weighting coefficients of the horizontally-polarized elements at $6.9 \mathrm{GHz}$ (weighting coefficients of the orthogonally-polarized elements are not shown due to their low values), in [dB]

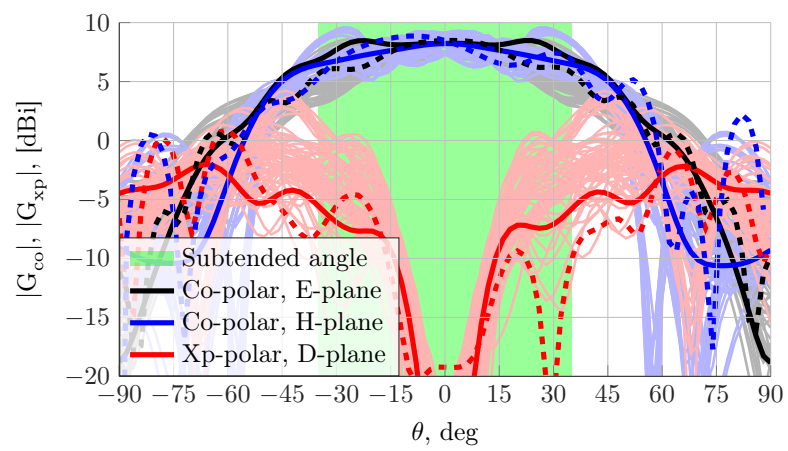

Fig. 4. (solid lines) The E-, H- and D-plane embedded element pattern (EEP) cuts of the 67-element array at C-band, simulated with the finite element method in HFSS software (reference case), where the bold lines denote the EEP of the central element (no. 18) of the full-size array, used for Simplified model I; and the dashed lines denote the EEP of the central element (no. 5) in the small-sized array, used for Simplified model II.

To cross-compare the array performances, we have used the active reflection coefficient [12] of the central element, when all antenna elements are excited with a certain complex-valued weight, as well as the radiometric characteristics specified in Table III.

The full-sized and small-scaled arrays have been modeled using a fullwave approach and the active reflection coefficient of the most excited elements are shown in Fig. 5. The red curve (a) corresponds to the fully-excited full-sized array; dashed curve (b) is for the same array when only 24 elements (highlighted in Fig. 3(a)) are active; and the blue curve (c) corresponds to the most excited element of the small array, when the same weight coefficients are used as for the previous case.

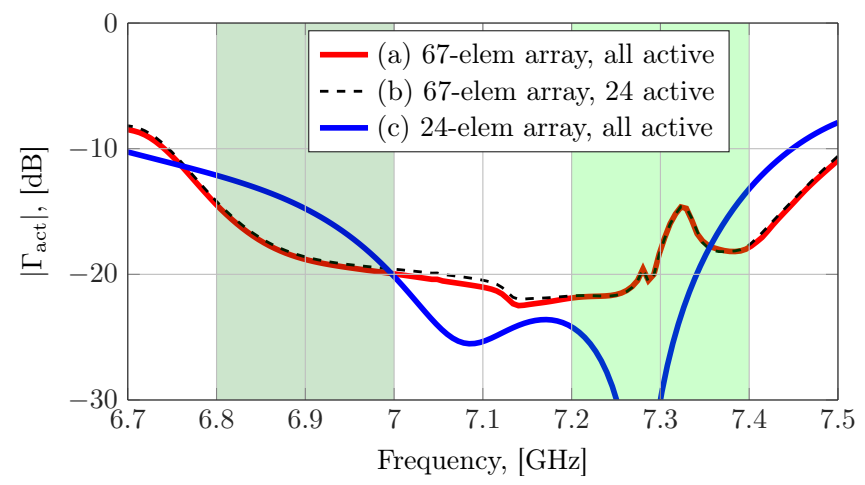

Fig. 5. Central active reflection coefficient for (a) full-size array, when all elements are excited to form the optimum beam; (b) full-size array, when only 24 most strongly excited elements are used in the calcultion; and (c) 24-element array with the same weight coefficients as for the previous case. The operating frequency bands are shown as green strips.

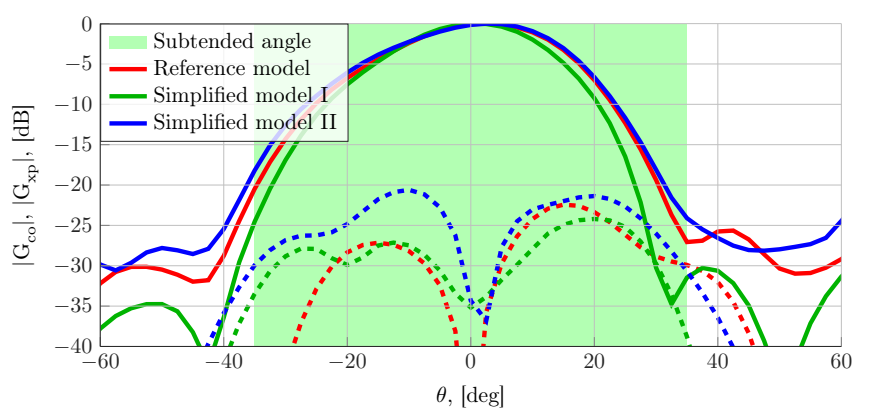

Fig. 6. Comparison of the total primary patterns obtained for the reference full-wave array model and Simplified models I and II. Solid and dashed lines show the co-polarized (at $\phi=0^{\circ}$ ) and cross-polarized (at $\phi=45^{\circ}$ ) field components, respectively.

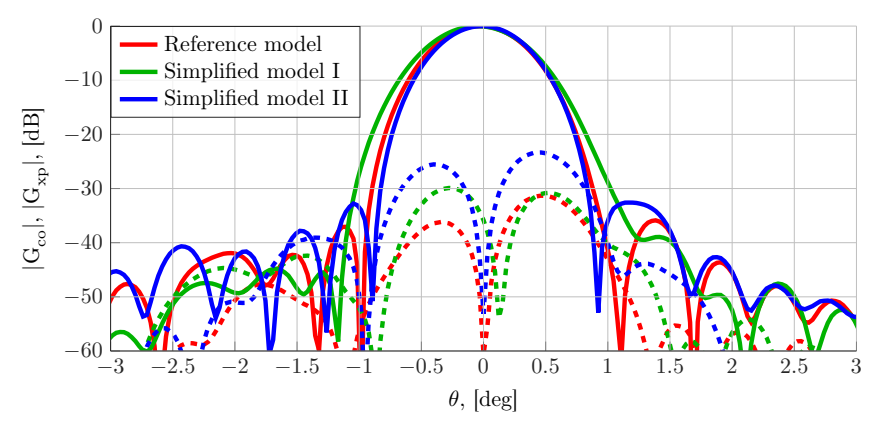

Fig. 7. Comparison of the total secondary patterns obtained for the reference full-wave array model and Simplified models I and II. Solid and dashed lines show the co-polarized (at $\phi=0^{\circ}$ ) and cross-polarized (at $\phi=45^{\circ}$ ) field components, respectively.

As one can see, the curves (a) and (b) are nearly identical. This is expected, since they are for the same EM model of the full-sized array, and the array elements outside the highlighted area are weakly excited, so they have neglifible effect on the central element active reflection coefficient. The result (c) differs from (b) since the edge truncation effects are stronger in the smaller array. Nevertheless, the overall prediction of the reference reflection coefficient (a) is good enough for such a 
TABLE III

FINAL RADIOMETER CHARACTERISTICS AT C-BAND (6.9 GHz)

\begin{tabular}{|l|c|c|c|c|}
\hline Radiometer characteristic & Requirement & Reference model & Simplified model I & Simplified model II \\
\hline Beam efficiency [\%] & & 96.6 & 97.8 & 96.5 \\
\hline Cross-polar. power, [\%] & $<0.34$ & 0.19 & 0.34 & 0.71 \\
\hline Distance to coast, [km] & $\leq 15$ & 11.4 & 14.5 & 13.6 \\
\hline Beam width, [deg] & & 0.648 & 0.664 & 0.647 \\
\hline Average footprint, [km] & 20 & 18.8 & 19.5 & 18.6 \\
\hline Footprint ellipticity & & 1.69 & 1.91 & 1.60 \\
\hline
\end{tabular}

strongly-coupled antenna array.

The total primary- and secondary patterns of the array, i.e. the pattern before and after reflection from the dish) are closscompared for the above cases in Fig. 6 and Fig. 7, respectively.

One can see the overall shape of the co-polar pattern of the reference full-wave array model has been predicted rather well with both simplified models, however the cross-polar components obtained with the latter appear to be higher. Similar observations can be made for the radiometric characteristics, cross-compared in Table III, where the distance to coast, beam width, footprint size and beam efficiencies have very similar values for all models, while the cross-polarization powers are a bit pessimistic for Simplified models I and II. More close investigation of the latter effects indicates the sensitivity of the presently used optimum beamforming approach to the variations and assymetries of the cross-polarization patterns. This will be studied in our future work.

\section{CONCLUSiOns}

The simplified modeling approach - assuming identical embedded element patterns of the phased array feed illuminating a large reflector - has been validated for the case of a conical scan radiometer antenna fed with a strongly coupled Vivaldi antenna element array. It has been shown that rather significant differences between the embedded element patterns, introduced by the edge truncation effects, have relatively weak contribution to the total compound beam of the array, when all elements are excited to provide optimum illumination. As the result, radiometer characteristics derived from the antenna far-field pattern, such as the beam efficiency, footprint, and distance to coast can be predicted almost as equally well as with the full-wave array model - that is important for the antenna system optimization and array prototype development phase. When applying this approach to applications with stringent requirements on the cross-poalrization, one could expect pessimistic estimation of its levels and the sensitivity to the optimum element excitation choice.

\section{ACKNOWLEDGMENT}

The present work has been funded by the Swedish National Space Board. The radiometer requirements have been derived by the team consisting of TICRA and DTU-Space (Denmark).

\section{REFERENCES}

[1] C. Bencivenni, M. Ivashina, R. Maaskant, and J. Wettergren, "Synthesis of maximally sparse arrays using compressive-sensing and full-wave analysis for global earth coverage," IEEE Trans. Antennas Propag., vol. PP, no. 99, pp. 1-6, 2016.

[2] L. Poli, P. Rocca, G. Gottardi, and A. Massa, "Design of simplified large array structures for preliminary experimental validation," in 10th European Conference on Antennas and Propagation (EuCAP), Davos, Switzerland, Apr. 2016, pp. 1-4.

[3] (2015) Earth Explorer Mission EE-9. [Online]. Available: http://explorercall.esa.int/index.php/15-mission-ee9

[4] F. Collard et al., "Sea surface temperature, wind and salinity (TWIST)," proposal for the Earth Explorer Mission EE-9, Unpublished.

[5] N. Skou and D. L. Vine, Microwave Radiometer Systems: Design \& Analysis. Artech House, 2006.

[6] C. Cappellin, K. Pontoppidan, P. Nielsen, N. Skou, S. S. Søbjærg, A. Ihle, D. Hartmann, M. Ivashina, O.Iupikov, and K. v. t Klooster, "Novel multi-beam radiometers for accurate ocean surveillance," in Proc. European Conference on Antennas and Propag. (EuCAP), The Hague, The Netherlands, Apr. 2014, pp. 1-4.

[7] O. A. Iupikov, M. V. Ivashina, C. Cappellin, and N. Skou, "Digitalbeamforming array antenna technologies for future ocean-observing satellite missions," in Proc. IEEE AP-S International Symposium, Fajardo PR, Jul. 2016, pp. 1-2.

[8] O. A. Iupikov, M. V. Ivashina, K. Pontoppidan, P. H. Nielsen, C. Cappellin, N. Skou, S. S. Søbjærg, A. Ihle, D. Hartmann, and K. v. t Klooster, "Dense focal plane arrays for pushbroom satellite radiometers," in Proc. European Conference on Antennas and Propag. (EuCAP), Hague, The Netherlands, Apr. 2014, pp. 1-5.

[9] C. Cappellin, K. Pontoppidan, P. H. Nielsen, N. Skou, S. S. Søbjærg, A. Ihle, M. V. Ivashina, O. A. Iupikov, and K. v. 't Klooster, "Design of a push-broom multi-beam radiometer for future ocean observations," in Proc. European Conference on Antennas and Propag. (EuCAP), Lisbon, Portugal, Apr. 2015, pp. 1-5.

[10] G. W. Kant, P. D. Patel, S. J. Wijnholds, M. Ruiter, and E. van der Wal, "EMBRACE: A multi-beam 20,000-element radio astronomical phased array antenna demonstrator," IEEE Trans. Antennas Propag., vol. 59, no. 6, pp. 1990-2003, Jun. 2011

[11] O. A. Iupikov, M. V. Ivashina, K. Pontoppidan, P. H. Nielsen, C. Cappellin, N. Skou, S. S. Søbjærg, A. Ihle, D. Hartmann, and K. v. t Klooster, "An optimal beamforming algorithm for phased-array antennas used in multi-beam spaceborne radiometers," in Proc. European Conference on Antennas and Propag. (EuCAP), Lisbon, Portugal, Apr. 2015, pp. 1-5.

[12] M. V. Ivashina, R. Maaskant, and B. Woestenburg, "Equivalent system representation to model the beam sensitivity of receiving antenna arrays," IEEE Antennas Wireless Propag. Lett., vol. 7, no. 1, pp. 733-737, Jan. 2008. 